\title{
QoS Restoration using a Disjoint Path Group in ATM Networks
}

\author{
K.H. Lee, Y.H. Choi, J.Y. Lee and S.B. Lee \\ Network and System Lab., Dept. of Electronics, Yonsei University, \\ 134 Shinchon-dong, Seodaemun-gu, 120-749, Seoul, Korea \\ Telephone: +8223612864. Fax: +8223124584 \\ E-mail: \{khlee, choi,jyl, sblee\}@nasla.yonsei.ac.kr
}

\begin{abstract}
A QoS restoration scheme for VP-based ATM network is proposed and its related characteristics are evaluated. The proposed scheme is a state-independent restoration scheme using a disjoint path group. A VP assignment and capacity planning problem using disjoint path group are formulated. When a failure occurs, failed working VPs are switched to protection paths with the same QoS level using a simple and fast restoration algorithm. The simulated restoration characteristics of the proposed algorithm are presented. A scheme of excess capacity assignment to protection path for dynamic traffic demands is also presented and analyzed.
\end{abstract}

\section{Keyword}

ATM, Survival Architecture and Performance, Protection Capacity Planning, QoS Restoration, Dynamic Traffic Demands

\section{Introduction}

To minimize the service disruption caused by link or node failures, many protection and restoration schemes have been presented and developed [1]. In particular, a restoration mechanism using ATM (Asynchronous Transfer Mode) and WDM (Wavelength Division Multiplexing) technology has been studied actively, and this technology will replace the current solution of SONET (Synchronous Optical Network) or SDH (Synchronous Digital Hierarchy)-based restoration mechanism. The advantage of the ATM restoration is that higher restoration throughput can be obtained at relatively low cost by the effective use of spare link capacity [2]. In addition, efficient QoS (Quality of Service) restoration is possible in ATM restoration [11]. In the future, WDM-based protection and restoration technology will be used more widely [1].

Restoration mechanism can be classified into several ways by which layer detects and performs restoration mechanism, what restoration algorithm is used between the restoration processing layer of each failed path, and what mechanism is prepared for enhancing the whole network restoration throughput. From many elements of restoration schemes, the most important thing is the fast restoration 
with simple restoration algorithm. This is because in current technology, the switching and restoration time of network facility is very low as compared to recommended restoration goal [1].

This paper introduces the protection and restoration scheme for VP (Virtual Path)-based ATM networks and evaluates a proposed protection scheme. For simple and fast restoration of failed connections, link disjoint or node disjoint VP groups (DVPG) are utilized here. More than two disjoint VP groups for the working and protection route are prepared. When a failure occurs, failed working VPs are switched to protection VPs of disjoint path groups with a simple restoration algorithm.

The rest of this paper is as follows. Section 2 introduces current ATM restoration architecture. In section 3, capacity planing problem with disjoint path group concepts is formulated. Restoration algorithm is discussed in section 4. In section 5, simulation results of proposed scheme are presented. A scheme for the variation of the traffic demands is presented in section 6 . Finally, section 7 concludes the paper.

\section{Network Survivability Architecture and Performance}

\subsection{Network survivability architecture}

High reliability of the transport function should be achieved to protect a network against failure and overload. Network survivability is defined as the set of network capabilities that allow a network to restore affected traffic in the event of a failure. Survivability architecture includes both protection switching and network restoration architecture type [3]. Protection makes use of pre-assigned capacity between nodes. Restoration makes use of any capacity available between nodes. In general, the algorithms used for restoration involve rerouting. In ITU-T I.311, these categories are further subdivided into protection switching, selfhealing and rerouting and these classes are defined as follows [4]:

- Protection switching: Protection switching is the establishment of a preassigned replacement connection automatically by means of equipment without the NMC (Network Management Center) function. The equipment may either reside in the connecting or terminating points of related VP-level.

- Rerouting: Rerouting is the establishment of a replacement connection by the NMC function. When a connection failure occurs, the replacement connection is routed depending on network resources available at that time.

- Selfhealing: Selfhealing is the establishment of a replacement connection by network without the NMC function. When a connection failure occurs, the replacement connection is found by the network elements and rerouted depending on network resources available at that time.

Protection and restoration scheme may be centralized or distributed by the control scheme. Restoration by distributed network control architecture is based on either management plane or control plane network protocols. In centralized net- 
work control architecture, restoration is accomplished by a network operation or management system. There are three general categories of topologies for protection architectures: point-to-point (linear), ring, and mesh. Protection switching is a distributed scheme used in linear or ring architecture. Selfhealing is a distributed scheme used in ring or mesh architecture. Rerouting is a centralized scheme used in mesh topology.

The protection network resource allocation policy refers to how network resource is allocated for protection usage. It may be dedicated or acquired ondemand from available resources. Two attributes of the protection connection are considered: route and bandwidth. There are three feasible policies that are considered [5].

- Dedicated: the route and bandwidth of the protection entity are pre-allocated. When a network impairment occurs, the network protection procedure is executed only at the end nodes supporting the protection entity.

- Semi-dedicated: Only the route of the protection entity is pre-allocated. Bandwidth of the protection entity is allocated from the available network capacity on a real-time basis. When a network impairment occurs, a bandwidth allocation procedure (e.g., under CAC procedure) is executed at the end nodes and all intermediate nodes supporting the protection entity.

- On-demand: The route and bandwidth of the protection entity are allocated from the available network resources on a real-time basis. When a network impairment occurs, a procedure for determining and allocating (e.g., under CAC procedures) the protection entity route and bandwidth from available network resources is executed by the network.

Protection involves the assignment of an alternative route with dedicated bandwidth assignment. In the event of a network impairment affecting the working route, a distributed management protocol is invoked to realize switchover. Restoration may be executed under the auspices of centralized control (reconfigurable networks), or it may involve either distributed control or management procedures (selfhealing networks). In both cases, resources may be semi-dedicated whereby the alternate route is predetermined but the bandwidth is assigned "ondemand" following fault detection, or both the route and the bandwidth may be assigned in real time ("on-demand"). The simplest protection architecture has one dedicated protection entity for each working entity. The most complex protection architecture has $\mathrm{m}$ protection entities shared amongst $\mathrm{n}$ working entities. When restoration is used, some percentage of the transport network capacity will be reserved for rerouting working traffic.

Depending on the location where traffic rerouting is performed, the restoration strategies can be categorized into three classes: local (line) restoration, regional (segment) restoration and end-to-end (path) restoration. When a node or link fails, the local restoration scheme dispatches alternate routes between the two neighbor nodes of the failed node or link, and reroutes all affected traffic around the failed node or link. In end-to-end restoration scheme, failed VPs are switched to alternate 
routes established between their respective source and destination nodes. In regional restoration scheme, alternate routes are searched in sub-network or segment where node or link failed. Local-to-end restoration scheme is another features of regional restoration scheme. End-to-end restoration scheme is more advantageous than local restoration in required spare capacity for restoration, but is more complex and exhaustive in network resources, and the restoration speed can be slow. Regional restoration scheme compromises all of these. Figure 1 illustrates local, regional, and end-to-end restoration example [6].

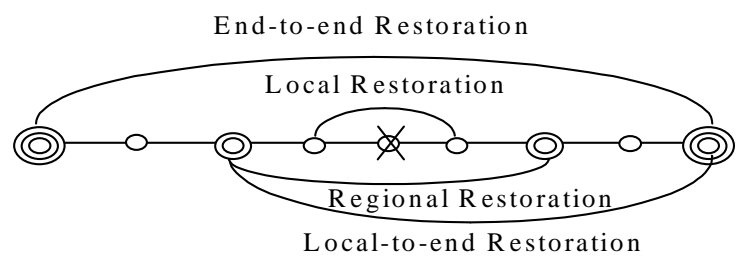

Figure 1: Restoration strategy

\subsection{Network protection performance}

From the user's perspective, an outage of the network can be represented by the following three major features: Unservability (U), Duration (D), Weight (W) [13]. Depending on the values of the $(\mathrm{U}, \mathrm{D}, \mathrm{W})$ triple, network outages may be classified as catastrophic, major, or minor. Survivability framework can be classified into physical, system, logical, and service layers by survivability technique and survivability measurement can be defined at each layer [7][13]. Performance objectives need to be defined for the ATM network survivability architectures and mechanisms. For more available and useful application, the performance of ATM network survivability architectures and mechanisms can be characterized in terms of defect/failure detection time, protection switching/restoration completion time, and restoration ratio (defined as the ratio of the restored working traffic to the impaired working traffic) [7].

- Network impairment detection indication time: This is the time it takes to detect and indicate the presence of a network impairment

- Network impairment confirmation time: This is the time it takes to confirm the persistence of the network impairment. This time allows lower layer protection actions to be completed

- Network Protection switching completion time: This is the time it takes to restore traffic (for a given protection capability) on the impaired entity to the protection entity once a decision to protect has been taken (i.e., at the end of the network impairment confirmation time).

- Network protection capability: The network protection capability refers to the percentage of impaired traffic that is to be protected within a given time.

Survivability requirements for a broadband network should be consistent with 
service availability requirements. Most services are impacted in the 2 to 10 seconds restoration time. For example, in the 2 to 10 seconds range, circuit switched calls may be dropped, private line and packet disconnects may be experienced, and data session time outs may occur. Restoration times will be more critical for some broadband services than others, but in general, data calls should not be dropped, retries should be avoided, lost data should be allowed to be recovered, and excessive retransmission should be avoided. Assuming that broadband networks will be supporting narrowband, high-speed, and other existing services via ATM, as well as new broadband applications, potential broadband network restoration times are recommended as listed in Table 1.

Table 1 Potential Broadband Network Recovery Time Target [5]

\begin{tabular}{|c|c|c|}
\hline Service Class & Time Targets & Restoration Times \\
\hline \hline Critical & 1 & $<50 \mathrm{~ms}$ \\
\hline \multirow{2}{*}{ Essential } & 2 & $50 \mathrm{~ms}<$ to $<200 \mathrm{~ms}$ \\
\cline { 2 - 3 } & 3 & $200 \mathrm{~ms}$ to $<2 \mathrm{sec}$ \\
\hline Basic & 4 & $2 \mathrm{sec}$ to $<10 \mathrm{sec}$ \\
\hline Economy & 5 & $10 \mathrm{sec}$ to $<5 \mathrm{~min}$ \\
\hline General & 6 & $>5 \mathrm{~min}$ \\
\hline
\end{tabular}

Critical services could include signaling, the transfer of monetary funds, and telemedicine applications. Interactive application which is sensitive to delay such as video teleconferencing may fall into essential service class. A VOD (Video On Demand) service that retrieves recorded video programs may be classified into a basic services. Services supporting e-mail or voice messaging could be classified as economy classes. General service is a category for traffic that is unprotected or traffic that is protected with mechanisms that have slower restoration times than the other service classes.

In current technology, equipment may not support restoration time target for all services. To meet the restoration goal, it is recommended that we must design simple algorithm with a minimum set of restoration messages, and enhance equipment performance by using a new technology such as parallel processing architectures. And, VPG (Virtual Path Group) concept may enhance the restoration time performance. VPG is a logical bundle of one or more ATM VP network and/or subnetwork connections that share the same transmission path(s) within the protected domain [8]. Also, it would be useful that different survival architecture is used based on the demand distribution, network connectivity, and demand growing conditions [1]. Considering the general performance of survival architecture classes, the protection or restoration speed is expected to be the fastest for the architectures with dedicated protection resources and slowest for the ones with on-demand protection resource allocation. Protection or restoration speeds for architectures with centralized control are expected to be slower than architectures with distributed 
control.

There can be exists that more than one restoration scheme is deployed. To accommodate the coexistence of multiple layers of restoration, an appropriate escalation strategy must be prepared. Other considerations beyond of these are often important to assess network survivability techniques. These are failure types for which network survivability technique can restore, amount of traffic rerouted with restoration process, required spare capacity for restoration. Restoration signal rate, message size, stability that is ability to predict the response of the restoration method to variations or perturbations in the network parameters, ability to interwork, implementation requirement are also important considerations [9].

\section{Working and Protection VP Capacity Assignment}

All VPs in networks have their own QoS classes. QoS parameters for ATM networks are Cell Delay Variation (CDV), Maximum Cell Transfer Delay (maxCTD), Cell Loss Ratio (CLR), Cell Error Ratio (CER), Maximum Cell Rate (maxCR), Available Cell Rate (AvCR), and Cell Rate Margin (CRM). All VCs (Virtual Channels) in a VP has same QoS classes in our network model. Each VP is restored with a VP having the same QoS level. The network is modeled to graph $G$ $=(N, L, C)$, where $N$ is the node set, $L$ is the link set, and $C$ is the cost. Let $\mathrm{Q}$ be the set of QoS with level 1 to $|\mathrm{Q}|$. If $P$ be the set of all VPs in the network, $P$ is then divided into $P=\left(P^{q}\right)$ according to QoS classes $q \in Q$.

Let $w=(s, d)$ in $W$ be a pair of nodes, $\pi_{\mathrm{w}}$ be the traffic demand of $w$ and $P_{w}$ be the path set for pair $w$. $\pi_{w}$ is composed of several traffic QoS demands $\pi_{w}=\left(\pi_{w}^{q}\right)$. Let $P_{w}^{q}$ be the path set for $\pi_{w}^{q}$, then $P_{w}^{q}$ is a subset of $P_{w}$ and $P^{q}$. These are also subsets of $P$. A path $p_{w, i}^{q}$ of $P_{w}^{q}$ is restored by another link or node disjoint path $p_{w, k}^{q}$ of $P_{w}^{q}$ for path index $i, k \in K(i \neq k) . P_{w}^{q}$ is divided into the disjoint path group $P_{w}^{u}=\left(P_{w}^{4, u}\right)$ where $a$ is an element of the set A with a value of 1 to $|\mathrm{A}|$ and has the property of $P_{w}^{q, a} \cap P_{w}^{q, b}=\Phi, \forall a, b \in A(a \neq b)$

Each link capacity is composed of a working capacity, a protection capacity, and an idle capacity. Each working and protection capacity is composed of multiple QoS traffic classes. We assume statistical multiplexing between same QoS classes, but no statistical multiplexing between diferrent QoS classes. But assuming unit cost function, the cost of each link is the sum of working and protection capacities. The problem of restoration network VP planning is the minimization of the total working and protection VP cost.

$$
\operatorname{Problem}(P)=\operatorname{Min} \sum_{l}\left(F_{l}+S_{l}\right)=\operatorname{Min} \sum_{l} \sum_{q}\left(F_{l}^{q}+S_{l}^{q}\right), \forall q \in Q, \forall l \in L \text { (1) }
$$

Subject to:

$$
F_{l}^{q}=\sum_{w \in W} \sum_{a \in A} \sum_{k \in K} f_{w, k}^{q} \cdot \alpha_{w, k}^{q, a} \cdot \phi_{w}^{q, a} \cdot \delta_{w, k, l}^{q}, \forall q \in Q, l \in L
$$




$$
\begin{aligned}
& S_{l}^{q} \geq \sum_{w \in W} \sum_{a \in A} \sum_{k \in K} f_{w, k}^{q} \cdot \alpha_{w, k}^{q, a} \cdot \varphi_{w}^{q, a} \cdot \delta_{w, k, l}^{q}, \forall q \in Q, \forall l \in L \\
& S_{l}^{q}+F_{l}^{q}=C_{l}^{q}, \sum_{q} C_{l}^{q} \leq C_{l}, \forall q \in Q, \forall l \in L \\
& \sum_{a \in A} \sum_{k \in K} f_{w, k}^{q} \cdot \alpha_{w, k}^{q, a} \cdot \phi_{w}^{q, a} \geq \pi_{w}^{q}, \forall q \in Q, \forall w \in W \\
& \sum_{a \in A} \sum_{k \in K} f_{w, k}^{q} \cdot \alpha_{w, k}^{q, a} \cdot \varphi_{w}^{q, a} \geq \pi_{w}^{q}, \forall q \in Q, \forall w \in W \\
& \sum_{a} \phi_{w}^{q, a}=1, \sum_{a} \varphi_{w}^{q, a}=|A|-1, \sum_{a} \phi_{w}^{q, a} \cdot \varphi_{w}^{q, a}=0, \forall q \in Q, \forall w \in W(7) \\
& \left(p_{w, i}^{q} \cdot \alpha_{w}^{q, a} \cdot \delta_{w, i, l}^{q}\right) \times\left(p_{w, k}^{q} \cdot \alpha_{w}^{q, b} \cdot \delta_{w, k, l}^{q}\right)=0, \\
& \forall w \in W, \forall a, b \in A(a \neq b), \forall i, k \in K(i \neq k), \forall l \in L \\
& \left(p_{w, i}^{q} \cdot \alpha_{w}^{q, a} \cdot \gamma_{w, i, n}^{q}\right) \times\left(p_{w, k}^{q} \cdot \alpha_{w}^{q, b} \cdot \gamma_{w, k, n}^{q}\right)=0 \text {, } \\
& \forall w \in W, \forall a, b \in A(a \neq b), \forall i, k \in K(i \neq k), \forall n \in N
\end{aligned}
$$

Equation (1) is the objective function for minimizing network working and protection costs. Equations (2) and (3) are the definition of working and spare capacities for each link $l$ and QoS class $q$, and equation (4) is link capacity constraints. Demand flow constraints for working and protection of each node pair $w$ and QoS Class $q$ are in (5) and (6). As can be seen from equation (7), working VPs are utilizing paths of one VP group among many defined path groups, and others are used for protection paths. Equations (8) and (9) represent link and node disjoint constraints.

This problem is a planning method of jointly considering working and protection cost at the same time. If we think only protection path or working path planning, we can obtain by considering only related parameter from above equation. Also, above problem is the case of path protection planning scheme. There can be exist other design formulation with line protection scheme, path protection without the disjoint constraints, and other schemes using released failed working capacity for other restoration path [10], or total reconfiguration of the entire network path [11]. 


\section{Restoration Algorithm}

\subsection{Basic Steps for Restoration}

As illustrated in Figure 2, network restoration can be accomplished through six basic steps in response to a network failure within the four survivability network layers:

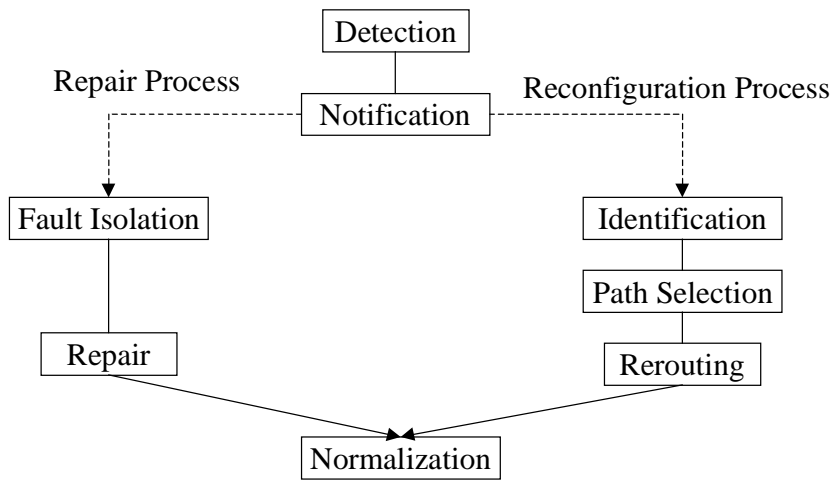

Figure 2: Restoration procedure [9]

When a link or node fails, neighbor node or NMC detects this. Notification of the network failure passed through the control architecture. Under a centralized scheme this means notifying the central operations support system or controller of the failure, either from the network element directly or via another operations support system for network surveillance. Under distributed control, this means notifying the other network elements via the links connecting network elements or a separate data communications network.

After the notification step, two parallel processes begin: (i) repair of the failed components, and (ii) reconfiguration of affected traffic. These steps can be applied to implementing most network survivability techniques, although some of the steps may not be necessary. Also note that some combinations of the steps may be iterated. For example, the path selection and rerouting steps may be iterated for each demand that is rerouted.

Under the repair process, the identification or location of the network failure is performed at fault isolation process. Next, the effort to repair the network failure to allow normalization of the network is performed.

Under the reconfiguration process, the affected demands are identified for rerouting. Then, path selection is performed by the selecting of an alternate path for each demand to be rerouted, either through data look-up or other algorithm. In flooding restoration scheme, restoration messages are overflowed and thus reduces the restoration throughput. Some efficient algorithms that minimize the message and achieve good results are presented [6][14]. Next, rerouting for the affected 
demands to an alternate path is performed.

Normalization is detection of the repair of the network failure and the return to the normal state of the network. This normalization could include rerouting of affected demands to new or original routes.

\subsection{Restoration algorithm for disjoint path group protection}

When a node or link fails, all paths passing this failed link or node must be recovered in a short time. When a signal fails to come in from a link, the link or neighboring node of that link would be failed. As soon as these failures are detected, the node sends a VP-AIS (Alarm Indication Signal) message to the destination ends of all VPs passing the failed link or node. When it receives this message, destination end node of failed working VP (dst) sends a restoration request message to the preassigned protection route. The intermediate node of this protection route processes this message and sends it to the next node of the protection route. In all intermediate nodes, resources for protection route are already prepared, and only set the exact VPI numbers and resources to the activated protection route, so the processing time can be minimal. If we can preserve protection VPI number with zero bandwidth as we can, it would be more useful. Finally, the source end node of failed working VP (src) processes the restoration request message and immediately switches the failed working path to this protection route. When sending cells to this new activated backup route, the VP end node sets a CLP (Cell Loss Priority) bit to 1 to avoid congestion when more link or node fails than that of prepared backup capability. When a congestive state exists in the network nodes, the cells with a CLP bit set are dropped first, therefore the network can exit from congestion immediately [2].

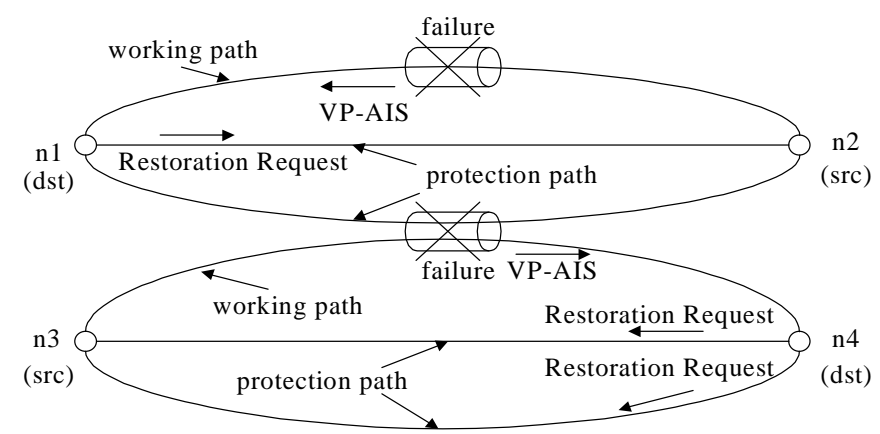

Figure 3: Restoration with 2 link failures $(|\mathrm{A}|=3)$

If the alternate disjoint path group numbers $(|\mathrm{A}|)$ are set to 2 in $\mathrm{VP}$ assignment problem, the network can be fully protected from one link or one node failure. In addition, if the alternate disjoint path group numbers are set to 3 like Figure 3, the network can be saved from simultaneous two links or two nodes failures. In this scheme, the end node of the failed path sends a restoration request message to the other two protection routes. The other end nodes switch the failed path to the pro- 
tection path in which the message arrived first or has better quality.

A proposed restoration scheme is a state-independent restoration scheme without considering the failure location by the use of a disjoint path group (DPG). The method of disjoint path groups for working and protection path increases the protection cost as compared to the state-dependent method. In the state-dependent restoration scheme, working and protection paths do not need to be fully link disjointed or node disjointed [12]. Many protection routes exist as with many failure scenarios and require more information and memory and more reserved VPI numbers. Identifying the failure location takes time varying on the implementation environment and technology. For simple and fast restoration, a link or node disjoint path group scheme has been chosen, and thus eliminates the consideration of any failure scenarios. In some cases, it would be more efficient if we switch all flow of one working group of one class of a source destination pair to the protection path, but the connections will experience out of synchronous in a short duration.

\section{Simulation Results of Proposed Restoration Algorithm}

The protection costs of various protection schemes were evaluated. The alternate disjoint group number of 2 and 3 for link failure protection and 2 for node failure protection were simulated. The simulated networks are grid and full mesh networks comprised of 3 to 64 nodes. The node disjoint protection scheme shows some $0-5 \%$ higher costs than the link disjoint protection scheme. Also, the link disjoint protection scheme with $|\mathrm{A}|=3$ shows approximately a $10-20 \%$ higher costs than the link disjoint scheme with $|\mathrm{A}|=2$.

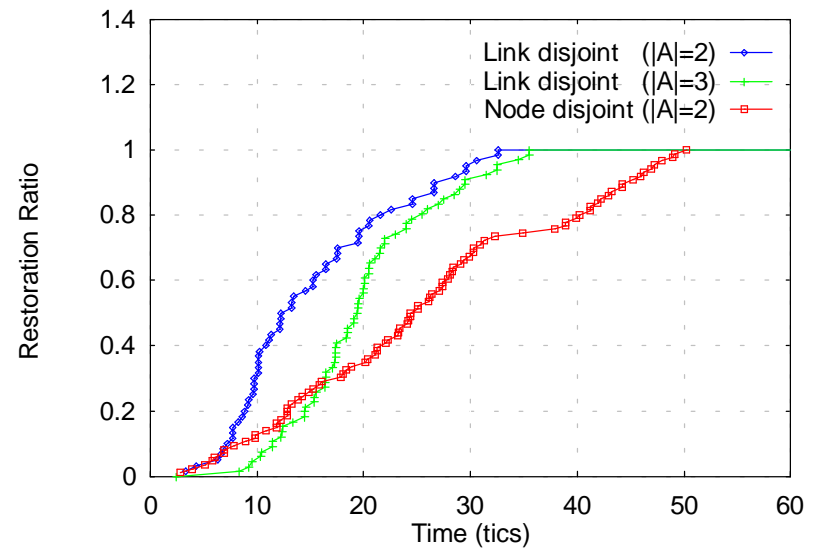

Figure 4: Restoration characteristics

The restoration characteristics of the proposed protection scheme with some protection alternatives were evaluated. The restoration characteristics of various scheme such as (a)link disjoint with $|\mathrm{A}|=2$, (b)link disjoint with $|\mathrm{A}|=3$, and (c)node disjoint with $|\mathrm{A}|=2$ are shown in Figure 4. Simulated network is a grid network with 25 nodes and 50 links. In this figure, failure type and number of failed VPs of 
each cases are (a)one link failure with failure of $40 \mathrm{VPs}$, (b)two link failure with failure of 66 VPs, and (c)one node failure with failure of 86 VPs. Processing time of one restoration message in a node is set to 1 unit (tics) in SLAMSYSTEM simulation packages. Restoration of failed VPs requires time for processing restoration messages. From this figure, one link failure case is faster than other two cases because total generated restoration message is small. Node failure case is the slowest because many failed VPs issues a lot of restoration messages. As the failed number of VPs increases, the number of generated restoration message increases, thus the network restoration time increases. So, when the numbers of failed VPs are same, the algorithm with smaller restoration messages will be faster. Reduction of the restoration messages reduces the network restoration time.

\section{Protection Capacity for Dynamic Traffic Demands}

When the traffic demand changes, the bandwidth of the working and protection path must be changed accordingly. The bandwidth is changed to maintain the cell level QoS in a VC and the blocking probability of the VCs in each VPs to the acceptable level [15][19]. In the pre-determined interval, restoration manager of NMC re-computes the changed traffic demand with call blocking probability, and reconfigures the route and capacity of the VPs in network. For maximizing network revenue under acceptable call blocking rate, we must prepare the scheme for the variation of network traffic demands. For traffic variation during the day, we can prepare several VP tables with each times for each SD pair [16]. Surely, protection path and capacity tables must be prepared with working VP tables. In our schemes using VPG concept, path and flow changes can be occurred more fluently because we can planned so that the traffic change occur within a VPG. Then, each node varies VP traffic as of load balancing scheme. Also, we must prepare traffic adaptation scheme between preplanned time intervals, for working and protection paths. There can be several schemes for this problem in our proposed disjoint protection scheme. High priority traffic can be recovered preferentially within a QoS class, or working traffics of one QoS class can preempt another class's protection bandwidth. But, we introduce 3 schemes not using such QoS preemption.

In scheme 1, only working capacity is changed with traffic variation and protection VPs are ignored and not changed. This scheme guarantees the original working traffic, but fails to recover additional increased traffic of the working VPs. After the next periods of reconfiguration by the NMC, the changed working VP traffic is protected by re-assigned protection VP. Scheme 2 is a method that guaranteeing the full restoration of the traffic of working VPs. For this, we search the route and the capacity of the working and protection path at the same time. When the capacity of the working VPs increases so that the blocking rate is over the acceptable level, working capacity is increased and a new protection VP for the overflowed demand is searched and found. The route of the protection VP must be disjoint with working VP. The capacity of the working VP is increased only when protection path with required capacity is found successfully. In this scheme, increasing cost is doubled as compared to first scheme. Scheme 3 is a method that 
excessively prepares protection bandwidth. When we assign protection bandwidth in assignment time, the protection capacity is assigned over the amount of the working capacity. As the working traffic increases, the protection path and capacity are unchanged until working capacity is increased to the pre-assigned protection capacity. When working capacity increases over the assigned capacity of the protection path, protection path and required capacity are searched as scheme 2 . At next re-assignment time by the NMC, protection capacity is re-assigned with excessively. So, this additional search of protection path and capacity can be minimized. We simulate these 3 different schemes.

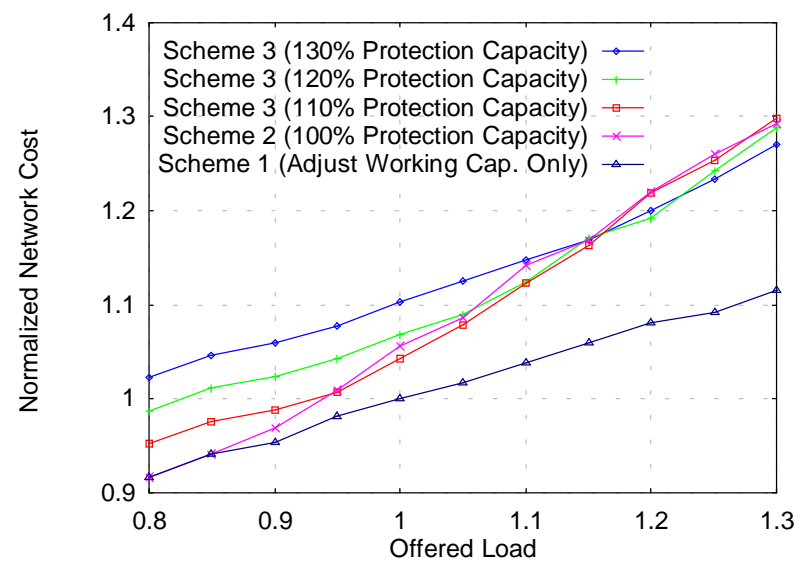

Figure 5: Normalized working and protection network costs.

Figure 5 is the simulation results. As the working traffic increases, the costs of the scheme 2 and scheme 3 increases faster than that of scheme 1. The meeting point of the two curves is different with the excess capacity ratio of the scheme 3 . This meeting point is below the preserved capacity ratio point. For example, the curve of scheme 3 with $120 \%$ protection capacity meet with the curve of scheme 2 at the input load point of 1.05 , and $130 \%$ is with the point of 1.15 . So, we can determine the required excess ratio of the preserved protection bandwidth by the characteristics of the variation of the network traffic demand. The main objective of the excess capacity assignment to protection path is to obtain lower control costs. Because protection costs is not increased linearly with the excess assignment ratio by the effect of sharing protection capacity, we can find the optimization points of suitable protection capacity.

\section{Conclusion}

A QoS restoration scheme for VP-based ATM networks using a fully disjoint link or node protection path group scheme was proposed. This scheme increases network cost somewhat, but the protection time can be reduced by the avoidance of failure identification time. If full disjoint path would be difficult to apply, partial disjoint path could be applied. Preparation of a multiple alternate disjoint path 
group scheme protects the cases of multiple failures but also increases network costs. A scheme of selectively applying alternate number can reduce the protection costs. QoS restoration is accomplished by preplanned protection path of same QoS class. Finally, dynamic bandwidth control with excess capacity assignment to protection VPs increases initial network costs, but achieves simplified bandwidth control and can be cost effective in some situation where traffic is increased monotonically and its variation is fast and large. Further, it would be required that network protection mechanism would be controlled by preparing restoration manager and well defined protection MIB (Management Information Base) [17][18].

\section{References}

[1] Tsong-Ho Wu, "Emerging Technologies for Fiber Network Survivability", IEEE Communications Magazine, February 1995

[2] Paul Veith and Dave Johnson, "ATM Network Resilience", IEEE Network, September/October 1997.

[3] ITU-T Recommendation G.805, "Generic functional architecture of transport networks", Geneva, November 1995.

[4] ITU-T Recommendation I.311, "B-ISDN general functional architecture", 1993.

[5] T1S1.5/96-065, "Broadband Service Availability", Bellcore, June 1996.

[6] K.H. Lee, Y.H. Choi, J.Y. Lee, and S.B. Lee, "Loop Expanding Fast Restoration", IEEE/IFIP NOMS '98, February 1998. New Orleans, Vol. 2, pp. 513-522.

[7] T1S1.5/96-071, "Broadband Protection Layer", Bellcore, June 1996.

[8] Jon Anderson, Curtis J. Newton, and Tai H. Noh, "Virtual Path Protection Switching-A Method for fast ATM Network Survivability", Bell Labs Technical Journal, Spring 1997, pp. 213-233

[9] T1 Technical Report No. 24, “A Technical Report on Network Survivability Performance", October 1993.

[10] B. Van Caenegen, N. Wauters and P. Dwmeester, "Spare Capacity Assignment for different Restoration Strategies an mesh survivable networks", IEEE Proceedings of ICC '97, pp. 287-292, 1997.

[11] T. Egawa, T. Komine, Y. Miyao,and F. Kubota, "QoS Restoration for Dependable Networks", IEEE/IFIP NOMS '98, February 1998, New Orleans, Vol. 2, pp. 503-512.

[12] Yijun Xiong and Lorne Mason, "On-state-independent and state-dependent path restoration in self-healing networks", IEEE Proceedings of ICC '98, Atlanta, Vol. 2, pp. 287-292.

[13] A. Zolfaghari and F. H. Kaudel, "A framework for Network Survivability Performance”, IEEE JASC, Vol. 12, No. 1, 1994., pp. 46-51.

[14] Hiroyuki Saito, Miroslaw M.Slominski and Makiko Yoshida, "An Improved Guided Restoration Algorithm for ATM Crossconnet Networks", IEEE/IFIP NOMS '96, Kyoto, Japan, Vol. 1, pp. 225-234.

[15] Ake Arvidsson, "Management of reconfigurable virtual path networks", 
Proceedings of $14^{\text {th }}$ Intl. Teletraffic Congress, 1994, pp. 931-940.

[16] D. Medhi, "Multi-Hour, Multi-Traffic Class Network Design for Virtual Path-Based Dynamically Reconfigurable Wide-Area ATM Networks", IEEE/ACM Tran. on Networking, Vol. 3, No. 6, Dec. 1995, pp. 809-818.

[17] K. H. Lee, Y. H. Choi, T. W. Kang, M. S. Do, J. Y. Lee and S. B. Lee, "Efficient Restoration Scheme for VP-based ATM Networks", Proceedings of APNOMS '97, Seoul, Korea, Oct. 1997, pp. 197-208.

[18] Tatsuyuki, KIMURA and Tatsuhiko YOSHIDA, "A Study on a Management Interface for VP Protection Switching”, IEEE Proceedings of $7^{\text {th }}$ ICCCN '98, Lafayette, Luisiana, Oct. 1998, pp. 539-546.

[19] Kazutaka Murakami and Hyong S. Kim, "Virtual Path Routing for Survivable ATM Networks", IEEE/ACM Tran. on Networking, Vol. 4, No. 1, Feb. 1996, pp. 22-39.

\section{Biographies}

Kil-Hung Lee received B.S. and M.S. degrees in Electronics Engineering from Yonsei University, Seoul Korea in 1989 and 1991 respectively. From 1980 to 1985, he engaged in Air Force Communication Sections. From 1991 to 1995, he engaged in LGIC (LG Information and Communication) for research and development engineer. He is a Ph.D student of Network Laboratory in Electronics at Yonsei University. He is a member of IEEE and has been working in AOW (Asia-Oceania working group) TG-NM (technical group of network management). His current research interests are computer networks, Internet and ATM network management.

Yong-Hoon Choi received B.S. and M.S. degrees in Electronics Engineering from Yonsei University in 1995 and 1997 respectively. He is a Ph. D. student in Yonsei University. He has been working in TG-NM. His current research interesta are ATM network management, distributed delegate and mobile agent in network management.

Jai-Yong Lee received the B.S degree in Electronic Engineering in 1977 from Yonsei University and M.S. and Ph.D. degrees in computer engineering in 1984 and 1987 respectively from Iowa State University. From 1977 to 1982, he was a research engineer at Agency for Defense Development of Korea. From 1987 to 1994 he was a Associate Professor at Pohang Institute of Science and Technology. $\mathrm{He}$ is currently a Professor in the Department of Electronic Engineering, Yonsei University. His current research interests are PCS, WATM and protocol testing.

Sang-Bae Lee received the B.S degree in 1961 from Seoul National University, the M.S degree from Stanford University in 1964 and the Ph.D degree from University Newcastle upon Tain in England in 1975. From 1969 to 1979, he was an assistant Professor in Seoul National University. He is a professor in Yonsei University since 1979. He was IEEE Korea Section chairman from 1986 to 1987, KITE vice chairman and chairman during the year 1989/1990. He was IEE Korea Section chairman in 1992. His research interests are in the area of computer network, data communication and circuit theory. 